\title{
(-)-Epigallocatechin-3-gallate attenuates myocardial injury induced by ischemia/reperfusion in diabetic rats and in H9c2 cells under hyperglycemic conditions
}

\author{
YANG WU ${ }^{1}$, ZHONG-YUAN XIA ${ }^{1}$, BO ZHAO $^{1}$, YAN LENG $^{1}$, JUAN DOU $^{2}$, \\ QING-TAO MENG ${ }^{1}$, SHAO-QING LEI ${ }^{1}$, ZHI-ZE CHEN $^{1}$ and JIE ZHU ${ }^{3,4}$ \\ Departments of ${ }^{1}$ Anesthesiology and ${ }^{2}$ Cardiac Surgery, Renmin Hospital of Wuhan University, \\ Wuhan, Hubei 430060; ${ }^{3}$ Department of Clinical Nutrition, Xin Hua Hospital \\ Affiliated to Shanghai Jiao Tong University School of Medicine; ${ }^{4}$ Department of Nutrition, \\ Shanghai Jiao Tong University School of Medicine, Shanghai 200092, P.R. China
}

Received May 26, 2016; Accepted May 23, 2017

DOI: 10.3892/ijmm.2017.3014

\begin{abstract}
Epigallocatechin gallate (EGCG) exerts multiple beneficial effects on cardiovascular performance. In this study, we aimed to examine the effects of EGCG on diabetic cardiomyopathy during myocardial ischemia/reperfusion (I/R) injury. EGCG (100 mg/kg/day) was administered at week 6 for 2 weeks to diabetic rats following the induction of type 1 diabetes by streptozotocin (STZ). At the end of week 8, the animals were subjected to myocardial I/R injury. The EGCG-elicited structural and
\end{abstract}

Correspondence to: Professor Zhong-Yuan Xia, Department of Anesthesiology, Renmin Hospital of Wuhan University, 238 Jiefang Road, Wuhan, Hubei 430060, P.R. China

E-mail: xiazhongyuanmz@aliyun.com

Dr Jie Zhu, Department of Nutrition, Shanghai Jiao Tong University School of Medicine, 1665 Kong Jiang Road, Shanghai 200092, P.R. China

Email: jacky284868@163.com

Abbreviations: AAR, area at risk; FoxO, Forkhead box O; CCA, common carotid artery; CON, control group; DM, streptozotocininduced diabetic rats; DMEM, Dulbecco's modified Eagle's medium; DMSO, dimethyl sulfoxide; +dp/dtmax, maximum speed of pressure development of left ventricle; -dp/dtmax, minimum speed of pressure decline of left ventricle; EGCG, (-)-epigallocatechin-3-gallate; EX, EX527; FBS, fetal bovine serum; H/R, hypoxia/re-oxygenation; HG, high glucose; I/R, ischemia/reperfusion; IS, infarct size; LAD, left anterior descending coronary artery; LDH, lactate dehydrogenase; LV, left ventricle; LVSP, left ventricular systolic pressure; MDA, malonaldehyde; MnSOD, manganese superoxide dismutase; NG, normal glucose; N, nomoxia; ROS, reactive oxygen species; STZ, streptozotocin; siRNA, small interfering RNA; SIRT1, silent information regulator 1; TTC, 2,3,5-triphenyl tetrazolium chloride; TUNEL, terminal deoxynucleotidyl transferase dUTP nick-end labeling; $15-\mathrm{F}_{2 \mathrm{t}}$-IsoP, $15-\mathrm{F}_{2 \mathrm{t}}$-isoprostane

Key words: diabetes, ischemia, reperfusion, (-)-epigallocatechin-3gallate, oxidative stress functional effects were analyzed. Additionally, EGCG $(20 \mu \mathrm{M})$ was administered for $24 \mathrm{~h}$ to cultured cardiac H9c2 cells under hyperglycemic conditions (30 $\mathrm{mM}$ glucose) prior to hypoxia/ reoxygenation $(\mathrm{H} / \mathrm{R})$ challenge, and its effects on oxidative stress were compared to $\mathrm{H} 9 \mathrm{c} 2$ cells transfecteed with silent information regulator 1 (SIRT1) small interfering RNA (siRNA). In rats with STZ-induced diabetes, EGCG treatment ameliorated postischemic cardiac dysfunction, decreased the myocardial infarct size, apoptosis and cardiac fibrosis, and reduced the elevated lactate dehydrogenase (LDH) and malonaldehyde (MDA) levels, and attenuated oxidative stress. Furthermore, EGCG significantly reduced H/R injury in cardiac H9c2 cells exposed to high glucose as evidenced by reduced apoptotic cell death and oxidative stress. The protein expression levels of SIRT1 and manganese superoxide dismutase (MnSOD) were reduced in the diabetic rats and the $\mathrm{H} 9 \mathrm{c} 2$ cells under hyperglycemic conditions, compared with the control rats following I/R injury and H9c2 cells under normal glucose conditions. EGCG pre-treatment significantly upregulated the levels of htese proteins in vitro and in vivo. However, treatment with EX527 and SIRT1 siRNA blocked the EGCG-mediated cardioprotective effects. Taken together, our data indicate that SIRT1 plays a critical role in the EGCG-mediated amelioration of I/R injury in diabetic rats, which suggests that EGCG may be a promising dietary supplement for the prevention of diabetic cardiomyopathy.

\section{Introduction}

Diabetic individuals are susceptible to ischemic heart disease and sustain a more unfavorable prognosis for survival than non-diabetic individuals, despite advancements in surgical techniques and pharmacological therapies (1). Hyperglycemia stimulates reactive oxygen species (ROS) production and induces oxidative stress (2), which is greater in the presence of diabetes mellitus following reperfusion injury, and contributes to the exacerbation of myocardial ischemia/reperfusion (I/R) injury (3). Furthermore, this condition has been evidenced to cause myocardial fibrosis and impair cardiac function in the left ventricle (LV) of diabetic rats (2). Therefore, the scavenging 
of ROS may effectively prevent the initiation or progression of diabetic myocardial I/R injury and heart failure $(2,4)$.

A recent finding suggests that there is a link between silent information regulator 1 (SIRT1) and the levels of ROS (5). As a type of histone deacetylase, SIRT1 plays a key role in a number of cell signaling pathways and widely regulates diverse biological processes, such as cell survival, apoptosis oxidative stress response and aging $(6,7)$. The activation of SIRT1 has been shown to significantly decrease ROS levels and promote cell survival (8). Furthermore, studies have shown that SIRT1 is an important cytoprotective and defensive cytokine against oxidative insults and the cardiac-specific overexpression of SIRT1 protects rats against myocardial I/R injury (9).

(-)-Epigallocatechin-3-gallate (EGCG) is a major bioactive polyphenol derived from green tea that has been found to possess potent antioxidant and free radical scavenging properties (10-12). It has been well documented that EGCG exerts multiple beneficial effects on cardiovascular performance, including reducing myocardial $\mathrm{I} / \mathrm{R}$ injury and alleviating post-ischemic myocardial dysfunction in vitro and in vivo $(11,12)$. Recent studies have indicated that some polyphenols reduce oxidative stress through the activation of SIRT1, thus resulting in the improvement of cardiac function in diabetic cardiomyopathy $(13,14)$. As an important polyphenol, EGCG may inhibit hyperglycemia-induced myocardial $\mathrm{I} / \mathrm{R}$ injury in diabetic hearts by a mechanism relative to the altered expression of SIRT1 and its downstream target genes.

Therefore, the present study aimed to evaluate the effects of EGCG treatment on cardiac function, myocardial apoptosis and oxidative stress, as well as its preventive role in the progression of cardiomyocyte injury under hyperglycemic conditions, as well as the effects of EGCG in diabetic rats subjected to myocardial I/R injury. In addition, we aimed to elucidate the possible mechanisms involving the altered expression of SIRT1 and its downstream target genes.

\section{Materials and methods}

Experimental animals and the induction of diabetes. All experimental protocols used in this study conformed to the guidelines for the Care and Use of Laboratory Animals published by the US National Institutes of Health (NIH Publication, revised 1996) and was approved by the Renmin Hospital of Wuhan University, Wuhan, China. Adult male Sprague-Dawley rats (weighing 270 $\pm 10 \mathrm{~g}$ ) were purchased from Beijing HFK Bioscience Co. (Beijing, China), as previously reported (15). The rats were allowed to acclimatize to a purified American Institute of Nutrition (AIN-93G) diet and distilled water ad libitum in the specific pathogen-free experimental animal facility of the Renmin Hospital for 5 days. Following acclimatization, the rats were randomly divided into the control group and the diabetic group. Type 1 diabetes was induced in the rats in the diabetic group by a single intraperitoneal injection of a freshly prepared streptozotocin (STZ) solution (Sigma-Aldrich, St. Louis, MO, USA) dissolved in $0.1 \mathrm{M}$ citrate buffer ( $\mathrm{pH} 4.5$ ) at a dose of $65 \mathrm{mg} / \mathrm{kg}$ body weight, as previously described (15). Rats in the control group received a single intraperitoneal injection of citrate buffer alone as a control. Three days post-STZ injection, tail vein blood glucose levels were measured using a One Touch Ultra Glucose meter (LifeScan, Milpitas, CA, USA) and rats with fasting blood glucose levels $>16.7 \mathrm{mM}$ were considered as diabetic (15). Body weight and serum glucose levels of the rats were recorded at the beginning of the experiment, and at 3 days, 6 and 8 weeks after the STZ-injection.

Animal experimental protocols. The animals were divided into 5 subgroups ( $\mathrm{n}=18$ for each subgroup) as follows: i) control rats (control); ii) diabetic rats (DM); iii) diabetic rats treated with EGCG (DM + EGCG); iv) diabetic rats treated with EGCG and the SIRT1 inhibitor, EX527 (Tocris Bioscience, Bristol, $\mathrm{UK})(\mathrm{DM}+\mathrm{EGCG}+\mathrm{EX})$; and v) diabetic rats treated with EX527 (DM + EX). EGCG ( $\geq 98 \%$ purity) was provided by Zhejiang Yixin Pharmaceutical Co., Ltd. (Zhejiang, China) (Cat no. 989-51-5). EGCG was dissolved in sterile-distilled deionized water as a stock solution of $10 \mathrm{mM}$ at $-80^{\circ} \mathrm{C}$ until dilution prior to use, as previously described $(16,17)$. EGCG was intragastrically administered to the rats for 14 consecutive days at a dose of $100 \mathrm{mg} / \mathrm{kg}$ body weight prior to the onset of myocardial I/R (17). EX527 was first dissolved in dimethyl sulfoxide (DMSO) and then diluted in sterile saline (final DMSO concentration <2\%). EX527 at a dose of $5 \mathrm{mg} / \mathrm{kg}$ was intraperitoneally injected every 2 days for 7 times prior to myocardial I/R operation. Rats in the control group were treated with sterile-distilled water by oral gavage (vehicle control for EGCG). Rats in the control, DM and DM + EGCG groups received a single injection of sterile saline with the final DMSO concentration $<2 \%$ respectively (vehicle control for EX527). The doses and time course of the experiments used for EGCG and EX527 in this study were based on published studies using the same animal species (12,17-19). After the treatment period, the diabetic and control rats were subjected to myocardial I/R operation. In the second set of experiments, 5 similar experimental groups of rats ( $n=7$ in each group) were subjected to the same experimental procedures and sacrificed at 14 days after I/R to assess myocardial fibrosis.

In vivo model of myocardial I/R injury. The animals were anesthetized by an intraperitoneal injection of sodium pentobarbital (65 mg/kg body weight) and then placed on a controlled heating pad to maintain rectal temperature at $37^{\circ} \mathrm{C}$, incubated and ventilated. The procedure for creating the myocardial I/R model was similar to that previously described (20). Briefly, a left thoracotomy was performed and the left anterior descending coronary artery (LAD) was ligated with 6-0 silk suture. The artery was occluded for $30 \mathrm{~min}$ by tightening the ligature. Following $30 \mathrm{~min}$ of ischemia, the ligature was loosened to allow reperfusion for $2 \mathrm{~h}$.

Hemodynamic detection. Invasive hemodynamic measurements were performed to evaluate I/R-induced cardiac dysfunction. Myocardial function was intermittently monitored at the baseline and after I/R insult. After the right common carotid artery (CCA) was separated, a polyethylene catheter was then advanced into the left ventricle via the incision on the right CCA. The catheter was connected to a pressure transducer (DPT-248; Yixinda, Shenzhen, China) and cardiac functional variables were recorded using LabChart 7 software (AD Instruments, Colorado Springs, CO, USA). Left ventricular systolic pressure (LVSP), maximum speed of pressure development of LV (+dp/dtmax) and pressure decline 
(-dp/dtmax) were continuously monitored using an electrophysiolograph (MH150; BioPAC, Goleta, CA, USA) at the time of $10 \mathrm{~min}$ prior to ischemia (baseline) and $2 \mathrm{~h}$ after reperfusion.

Assessment of myocardial injury. To assess the levels of lactate dehydrogenase (LDH), arterial blood samples were collected at the end of reperfusion and centrifuged at 2,000 $\mathrm{xg}$ for $10 \mathrm{~min}$ to collect serum. Levels of serum LDH were detected using a commercial enzyme-linked immunosorbent assay (ELISA) kit (Nanjing Jiancheng Bioengineering Institute, Nanjing, China) according to the manufacturer's instructions.

Determination of apoptosis and myocardial infarction. At the end of the $2 \mathrm{~h}$ of reperfusion, the hearts were rapidly removed and rinsed in ice-cold saline and then embedded in optimal cutting temperature compound, followed by being frozen at $-80^{\circ} \mathrm{C}$ for cryosectioning. Myocardial apoptosis was analyzed by terminal deoxynucleotidyl transferase dUTP nick-end labeling (TUNEL) reaction using an in situ cell death detection kit (Roche Diagnostics GmbH, Mannheim, Germany) as previously described (20). For each heart sample, a total of 15 random microscopy fields were selected and the index of apoptosis was calculated as a percentage of apoptotic myocytes to the total number of myocytes. The infarct size (IS) and area at risk (AAR) were measured using Evans blue dye (2\% EB; Sigma-Aldrich) and 2,3,5-triphenylte trazolium chloride (1\% TTC; Sigma-Aldrich) staining. At the end of reperfusion, the ligature around the coronary artery was retired again and $1 \mathrm{ml}$ of $2 \% \mathrm{~EB}$ was injected into the aorta. The presence of EB was used to identify the area that was not subjected to ischemia. The rats were euthanized and the hearts were rapidly excised and frozen at $-20^{\circ} \mathrm{C}$, and then sliced into 2-mm-thick sections parallel to the atrioventricular groove using a heart slice chamber. The slices were incubated in $1 \%$ TTC in buffer ( $\mathrm{pH} \mathrm{7.4)} \mathrm{for} 15 \mathrm{~min}$ at $37^{\circ} \mathrm{C}$. The viable tissue was stained red by TTC, while the infarct portion not taking up TTC stain remained pale. Morphometric measurements of the area at AAR and IS in each slice were performed as previously described (20). The percentage of ratios of AAR vs. LV (AAR/ LV) and IS vs. AAR (IS/AAR) were calculated.

Masson's trichrome staining. To objectively quantify the amount of tissue fibrosis, another subset of rats $(=35)$ were euthanized 14 days after I/R. Their hearts were rapidly removed and incubated with $10 \%$ formalin overnight at room temperature overnight, and embedded with paraffin. The hearts were sliced horizontally to the long axis, and stained with hematoxylin and eosin, and Masson's trichrome stain for light microscopy examinations. Masson's trichrome staining was performed using a Masson Stain kit (D026; Nanjing Jiancheng Bioengineering Institute, Nanjing, China). Digital images were obtained at 400 magnification by microscopy (Olympus, Tokyo, Japan). Fifteen randomly selected microscopic fields from Masson's trichrome-stained sections were analyzed. The percentage of fibrosis was determined using ImageJ software (NIH, Bethesda, MD, USA) to quantify blue (fibrotic) vs. non-blue (non-fibrotic) areas.

Determination of free $15-F_{2 t}$-isoprostane $\left(15-F_{2 t}-I\right.$ soP) and malonaldehyde content. Myocardial tissue from the left ventricular ischemic region was homogenized immediately after reperfusion. As a specific indicator of oxidative stress, free $15-\mathrm{F}_{2 \mathrm{t}}$-IsoP in the homogenized heart tissue was determined using commercially available kits (Cayman Chemical Co., Ann Arbor, MI, USA). The level of malonaldehyde (MDA) from the homogenized heart tissue and culture mediaum were measured using respective commercial kits (Nanjing Jiancheng Bioengineering Institute, Nanjing, China) according to the manufacturer's instructions. The results were expressed as $\mathrm{mg} / \mathrm{g}$ protein for $15-\mathrm{F}_{2 \mathrm{t}}$-IsoP and $\mathrm{nmol} / \mathrm{mg}$ protein for MDA.

Cell culture and establishment of a hypoxia/reoxygenation $(H / R)$ model. Rat myocardium-derived H9c2 cells were obtained from the American Type Culture Collection (ATCC, Manassas, VA, USA) and maintained in Dulbecco's modified Eagle's medium (DMEM) containing 10\% fetal bovine serum (FBS), $100 \mathrm{U} / \mathrm{ml}$ penicillin and $100 \mu \mathrm{g} / \mathrm{ml}$ streptomycin at $37^{\circ} \mathrm{C}$ in a humidified atmosphere with $5 \% \mathrm{CO}_{2}$ and $95 \%$ air. DMEM containing $30 \mathrm{mM}$ glucose has been applied to simulate chronic hyperglycemia in cell-based studies (15). The H9c2 cells in our study were cultured in DMEM containing $5.5 \mathrm{mM}$ glucose [normal glucose (NG)] or $30 \mathrm{mM}$ glucose [high glucose (HG)]. Following exposure to normal glucose or high glucose medium for $48 \mathrm{~h}$, the cells were randomly assigned to 5 groups as follows: i) normal glucose $(\mathrm{NG})$ medium $+\mathrm{H} / \mathrm{R}$; ii) high glucose $\mathrm{HG}$ medium $+\mathrm{H} / \mathrm{R}$; iii) $\mathrm{HG}+\mathrm{EGCG}+\mathrm{H} / \mathrm{R}$; iv) $\mathrm{HG}+\mathrm{EGCG}+\mathrm{SIRT} 1$ small interfering RNA (siRNA) + H/R; v) $\mathrm{HG}+\mathrm{EGCG}+$ scramble siRNA + H/R. The cells were treated with EGCG and/or siRNA $24 \mathrm{~h}$ prior to the H/R challenge. For the induction of $\mathrm{H} / \mathrm{R}$ injury, hypoxic conditions were obtained by a three gas incubator containing $94 \% \mathrm{~N}_{2}$ and $5 \% \mathrm{CO}_{2}$. Cardiomyocytes were subjected to $\mathrm{H} / \mathrm{R}$ by hypoxia for $2 \mathrm{~h}$ followed by $4 \mathrm{~h}$ of reoxygenation in all groups. EGCG was freshly prepared as a stock solution of $10 \mathrm{mM}$ by dissolving the compound in deionized water (12) and added to culture medium at a concentration of $20 \mu \mathrm{M}$ prior to $\mathrm{H} / \mathrm{R}$. Scramble siRNA or SIRT1 siRNA (sc-108043; Santa Cruz Biotechnology, Inc., Santa Cruz, CA, USA) were transfected into the cells $24 \mathrm{~h}$ prior to $\mathrm{H} / \mathrm{R}$ challenge according to the manufacturer's instructions.

Measurement of cell viability and LDH activity. After the treatments, cell viability was assessed by using 3-[4, 5-dimethylthiazol- 2-yl]-2,5-diphenyltetrazolium bromide (MTT; Beyotime Biotechnology Institution, Jiangsu, China) according to the manufacturer's instructions. Cardiomyocyte injury was assessed by measuring LDH release into the culture medium with an LDH activity assay kit (Beyotime, Haimen, China). The LDH concentration was measured in the medium using a spectrophotometer set at $450 \mathrm{~nm}$.

Assessment of cell apoptosis by flow cytometric analysis. After the completion of the various treatments, the cells were collected and resuspended in binding buffer. Following the additioin of fluorescein isothiocyanate (FITC)-conjugated Annexin V and propidium iodide (PI), cellular fluorescence was measured on a FACSCalibur and data obtained was analyzed with CellQuest Pro software (BD Biosciences, Franklin Lakes, NJ, USA).

Western blot analysis. Equal amount of proteins from rat hearts and $\mathrm{H} 9 \mathrm{c} 2$ cell homogenate were separated by electrophoresis 
and transferred to polyvinylidene difluoride membranes (Millipore, Billerica, MA, USA). Each membrane was incubated overnight at $4^{\circ} \mathrm{C}$ with the following specific primary antibodies: SIRT1 (Rab. sc-15404, 1:500 in blocking buffer in TBS) and MnSOD (Rab. sc-137254, 1:500 in blocking buffer in TBS) (all from Santa Cruz Biotechnology, Inc.). The membranes were then incubated with secondary antibody $(1: 10,000$; IRDye $800 \mathrm{CW}$; LI-COR Corporate, Lincoln, NE, USA) for $1 \mathrm{~h}$ at room temperature. Immune complexes were visualized by fluorescence imaging scanner (LI-COR Corporate). Band densities were quantified and calculated by Odyssey Image Analysis software (LI-COR Corporate) and then normalized to glyceraldehyde 3-phosphate dehydrogenase (GAPDH). The protein expression amounts were represented relative to those of the control.

Statistical analysis. All data were expressed as the means \pm SEM and analyzed using GraphPad Prism 6 statistic software (GraphPad Software, Inc., La Jolla, CA, USA). Differences between the groups were analyzed by using a one-way ANOVA with Tukey's test. Unpaired two-tailed Student's t-test was applied for the comparison between two different groups. The changes in body weight, serum glucose levels and systemic hemodynamics over time were analyzed via a two-way ANOVA with Bonferroni's test. The values at $\mathrm{P}$-value $<0.05$ were considered statistically significant.

\section{Results}

A total of 125 rats were used in our experiments: 7 rats died during the experimental intervention of ischemia for $30 \mathrm{~min}$ (1 in the control group, 2 in the DM group, 1 in the DM + EGCG group, 2 in the DM + EGCG + EX group, and 1 in the DM + EX group) and 7 rats died during the experimental intervention of reperfusion for $2 \mathrm{~h}$ (1 in the control group, 2 in the DM group, 1 in the DM + EGCG group, 1 in the DM + EGCG + EX group and 2 in the DM + EX group). Right after I/R intervention, the rats from each group were sacrificed for the experiments of hemodynamic detection, apoptosis, infarct size, MDA and western blot analysis. Additionally, 6 rats died during 14 days after I/R administration ( 2 in the DM group, one in the DM + EGCG group, one in the DM + EGCG + EX group and 2 in the DM + EX group). Fourteen days after I/R administration, in order to assess myocardial fibrosis, 5 surviving rats from each group were adopted and then sacrificed for Masson's trichrome staining (29 rats). Data were reported on the remaining 105 rats.

Characteristics of control and diabetic rats. As shown in Fig. 1, initial body weight and serum glucose levels were similar between all groups $(\mathrm{P}>0.05)$. The diabetic rats exhibited a marked increase in serum glucose levels and a decrease in body weight following the induction of diabetes $(\mathrm{P}<0.001$ vs. control). EGCG and EX treatment did significantly alter these levels in the diabetic rats, as compared to the vehicle-treated diabetics $(\mathrm{P}>0.05)$.

Status of systemic hemodynamics in control and diabetic rats. The serial changes in LV hemodynamic parameters, including LVSP, $+\mathrm{dp} / \mathrm{dtmax}$ and $-\mathrm{dp} / \mathrm{dtmax}$ during the experiments, are
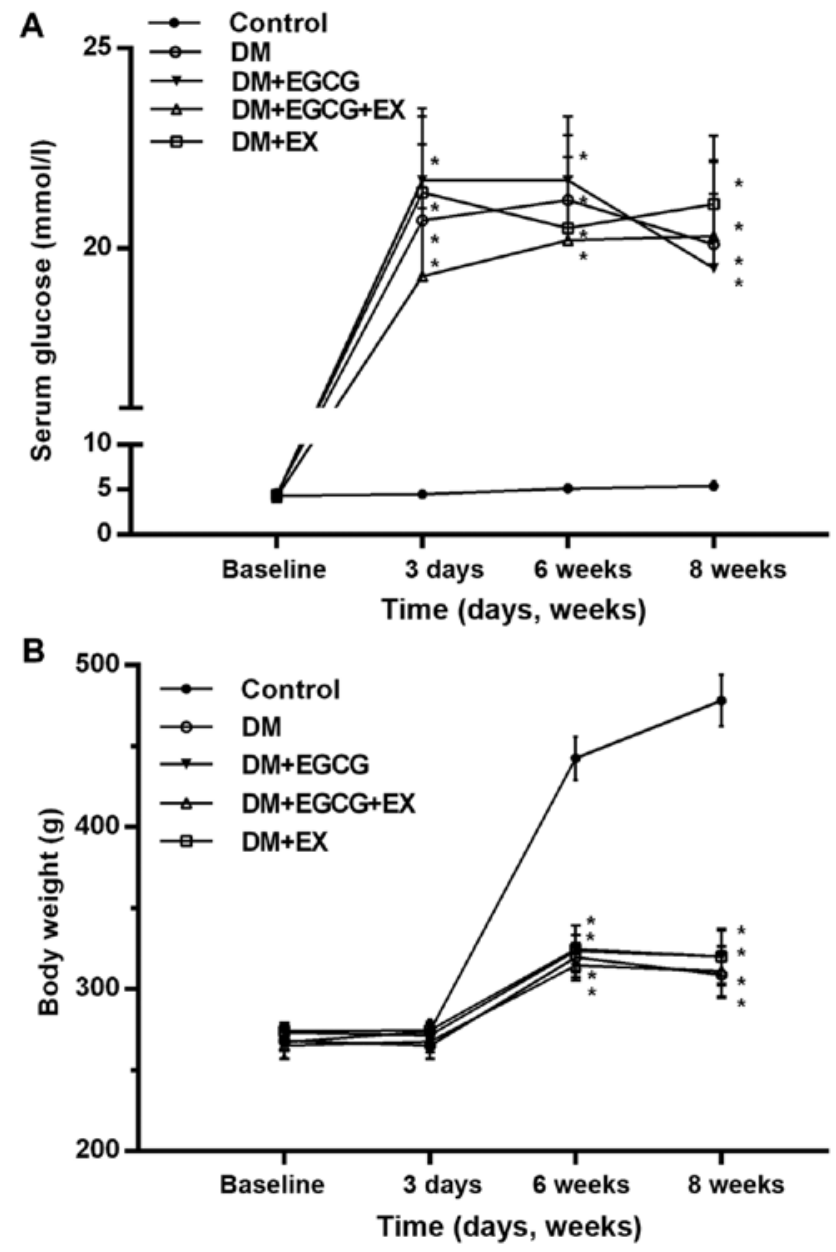

Figure 1. Body weight and serum glucose concentrations in control and diabetic rats. (A) Serum glucose concentrations and (B) body weights in control and streptozotocin-induced diabetic rats that were treated with or without (-)-epigallocatechin gallate (EGCG). Values are the means \pm SEM, $n=18$. ${ }^{*} \mathrm{P}<0.05$ vs. control group.

shown in Fig. 2. At baseline, the LVSP, $+\mathrm{dP} / \mathrm{dtmax}$ and $-\mathrm{dP} /$ dtmax in all diabetic groups were lower than those in the control group (all $\mathrm{P}<0.05$ ) (Fig. 2). There were no LV function differences between the diabetic groups at baseline $(\mathrm{P}>0.05)$. At the time point of I30, R60 min and R120 min, a significant increase in LVSP, $+\mathrm{dP} / \mathrm{dtmax}$ and $-\mathrm{dP} / \mathrm{dtmax}$ was observed in the DM + EGCG group $(\mathrm{P}<0.05)$, as compared to the DM group, although the respective values were still lower than those in the control group $(\mathrm{P}<0.05)$. However, the improvements in LV function induced by EGCG were not observed in the $\mathrm{DM}+\mathrm{EGCG}+\mathrm{EX}$ group $(\mathrm{P}<0.05)$.

Myocardial infarct size, cardiomyocyte apoptosis and serum LDH level in control and diabetic rats. Compared with the control group, the infarct size, cardiomyocyte apoptosis and serum LDH levels significantly increased in the DM group (all $\mathrm{P}<0.05$ ) (Figs. 3 and 4), whereas the EGCG administration significantly decreased the infarct size (Fig. 3A and B), cardiomyocyte apoptosis (Fig. 3C) and serum LDH levels (Fig. 4A) by $17.8,22.7$ and $27.7 \%$, respectively (all $\mathrm{P}<0.05$ ). However, the protective effects of EGCG were abolished by concomitant treatment with EGCG and EX527, with the infarct size, rate of apoptosis and serum LDH levels increasing by $25.6,30.5$ 

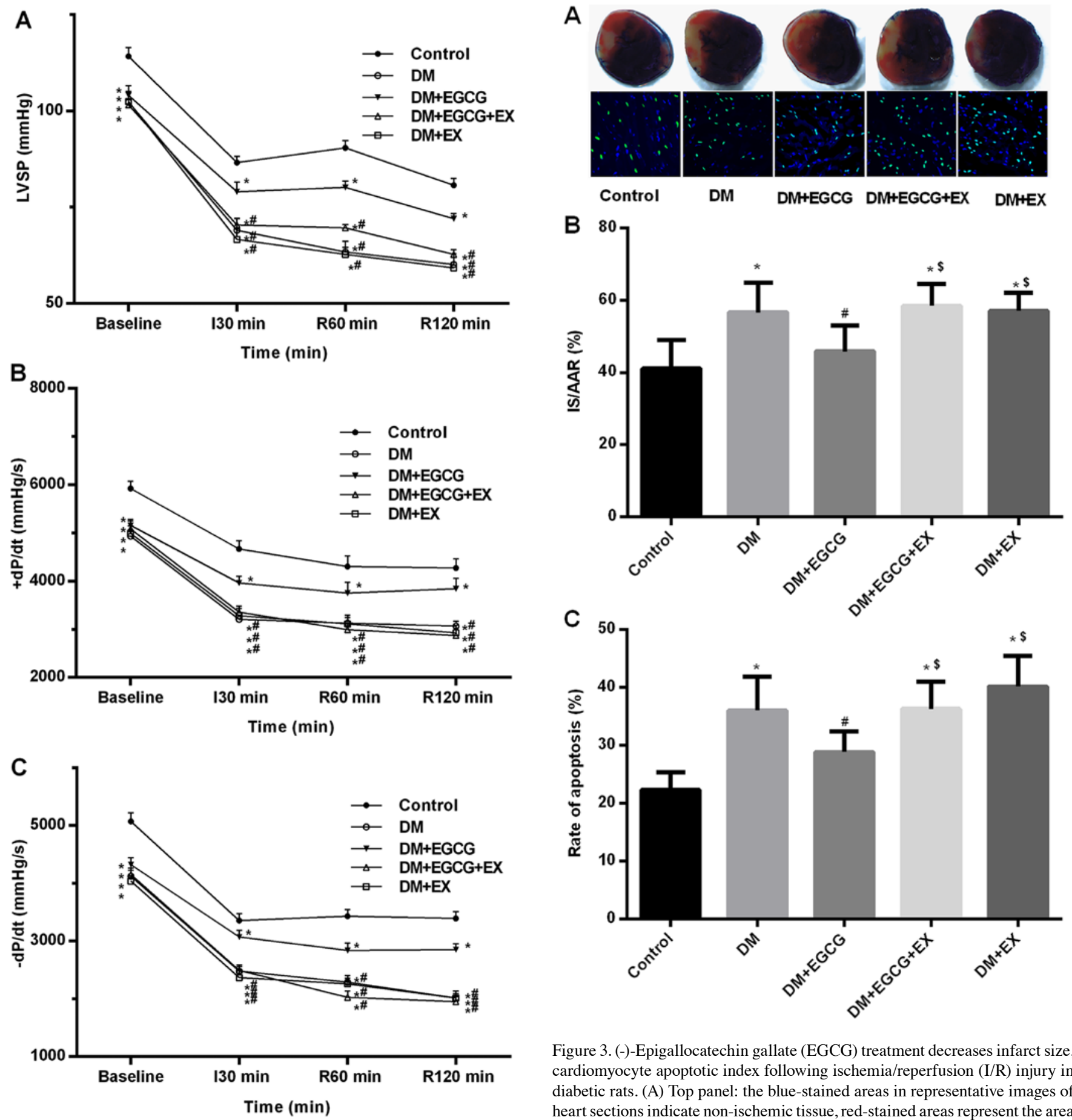

Figure 3. (-)-Epigallocatechin gallate (EGCG) treatment decreases infarct size, cardiomyocyte apoptotic index following ischemia/reperfusion (I/R) injury in diabetic rats. (A) Top panel: the blue-stained areas in representative images of heart sections indicate non-ischemic tissue, red-stained areas represent the area at risk and negative-stained areas indicat infarct areas. Bottom panel: images of the apoptotic myocytes are shown by TUNEL staining; green fluorescence shows TUNEL-positive nuclei; blue fluorescence shows nuclei of total cardiomyocytes. Images are at x 400 magnification; a total of 15 fields/heart were selected. (B) Myocardial infarct size was expressed as a percentage of the area at risk (AAR) $(\mathrm{n}=6)$; (C) Apoptotic index was expressed as a percentage of TUNEL-positive cells $(\mathrm{n}=6)$. Values are the means \pm SEM. ${ }^{\mathrm{P}}<0.05$ vs. control group; ${ }^{\#} \mathrm{P}<0.05$ vs. $\mathrm{DM}$ group; ${ }^{\$} \mathrm{P}<0.05$ vs. $\mathrm{DM}+\mathrm{EGCG}$ group.

and $38.9 \%$, respectively (all $\mathrm{P}<0.05$ ), compared to the diabetic group treated with EGCG alone (Figs. 3 and 4).

Myocardial $15-F_{2 t}$ formation and MDA levels in control and diabetic rats. Following $2 \mathrm{~h}$ of reperfusion, $15-\mathrm{F}_{2 \mathrm{t}}$ formation and MDA levels in the diabetic rats were higher than those in the control group (152.6 and $165.8 \%$, respectively, both $\mathrm{P}<0.05$ ) (Fig. 4B and C). However, EGCG treatment reduced

$15-\mathrm{F}_{2 \mathrm{t}}$ and MDA formation by 21.4 and $24.9 \%$ in the diabetic rats following myocardial I/R injury, compared to those in the DM group $(\mathrm{P}<0.05)$; these effects were abrogated by the administration of EX527 (Fig. 4B and C).

Protein expression of SIRT1 and MnSOD in control and diabetic rats. As shown in Fig. 5, STZ-induced diabetes reduced 

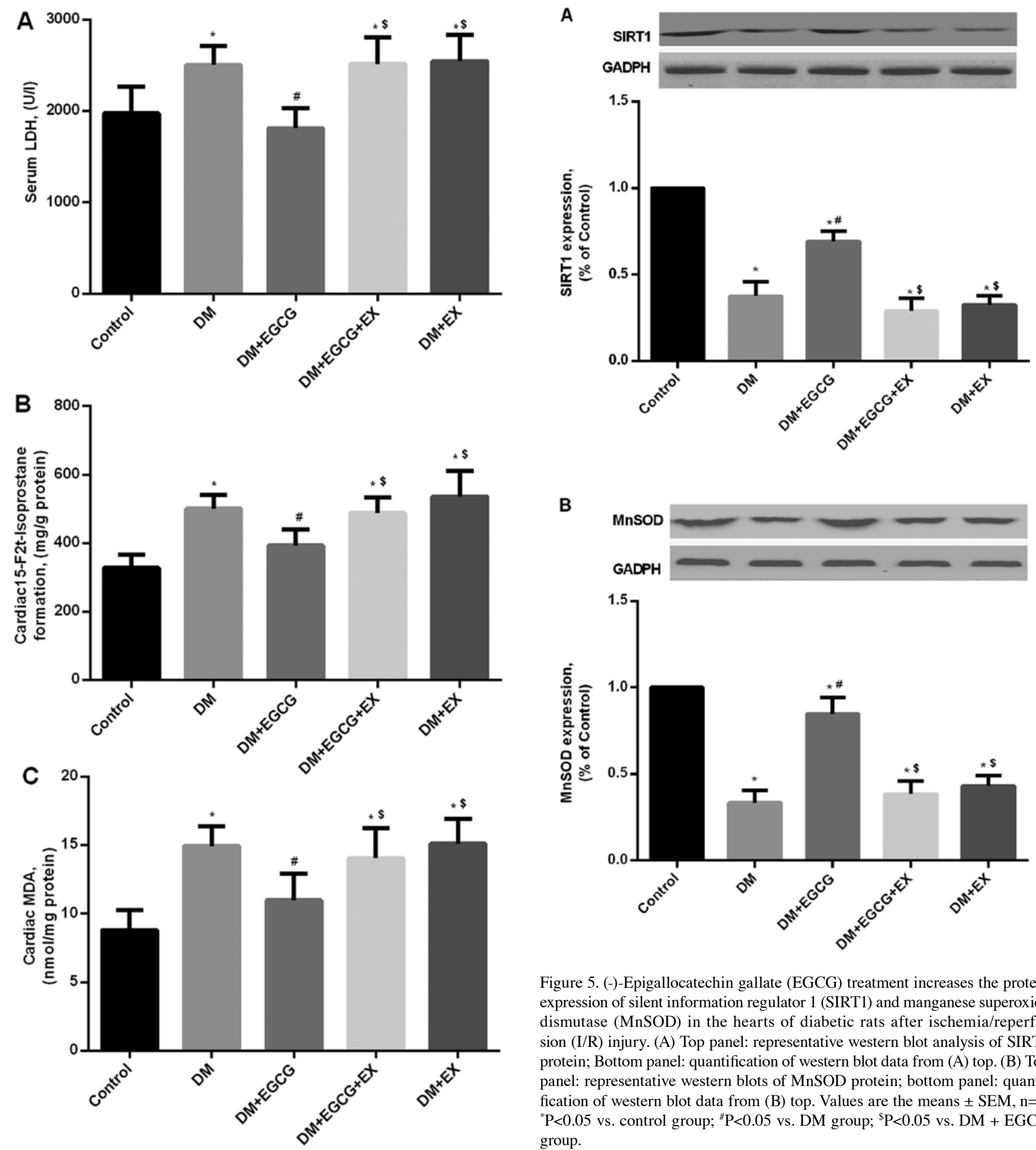

Figure 5. (-)-Epigallocatechin gallate (EGCG) treatment increases the protein expression of silent information regulator 1 (SIRT1) and manganese superoxide dismutase (MnSOD) in the hearts of diabetic rats after ischemia/reperfusion (I/R) injury. (A) Top panel: representative western blot analysis of SIRT1 protein; Bottom panel: quantification of western blot data from (A) top. (B) Top panel: representative western blots of MnSOD protein; bottom panel: quantification of western blot data from (B) top. Values are the means \pm SEM, $n=6$. ${ }^{2} \mathrm{P}<0.05$ vs. control group; ${ }^{\text {"P }}<0.05$ vs. DM group; ${ }^{\$} \mathrm{P}<0.05$ vs. DM + EGCG group.

Figure 4. (-)-Epigallocatechin gallate (EGCG) treatment decreases serum lactate dehydrogenase (LDH), cardiac malonaldehyde (MDA) content and $15-\mathrm{F}_{2 \mathrm{t}}$-isoprostane formation following ischemia/reperfusion ( $\left.\mathrm{I} / \mathrm{R}\right)$ injury in diabetic rats. (A) Serum LDH level, (B) the level of $15-\mathrm{F}_{2 t}$-isoprostane and (C) MDA contents in cardiomyocytes were compared among different groups $(\mathrm{n}=6)$. Values are the means $\pm \mathrm{SEM}$. ${ }^{*} \mathrm{P}<0.05$ vs. control group; ${ }^{\#} \mathrm{P}<0.05$ vs. DM group; ${ }^{\mathrm{P}} \mathrm{P}<0.05$ vs. $\mathrm{DM}+\mathrm{EGCG}$ group.

the expression of SIRT1 protein in the DM group $(\mathrm{P}<0.05)$, compared with the age-matched control group following $\mathrm{I} / \mathrm{R}$ injury, whereas EGCG pre-treatment significantly increased SIRT1 expression compared to the DM group $(\mathrm{P}<0.05)$ (Fig. 5A). EGCG did not promote the increased expression of SIRT1 in the

diabetic rats in the presence of EX527 pre-treatment $(\mathrm{P}>0.05)$, compared with the DM + EGCG group (Fig. 5A). Additionally, a higher level of MnSOD expression was also found in the EGCG pre-treatment group, but the increment was abolished by EX527 intervention $(\mathrm{P}<0.05)$, compared to the DM + EGCG group (Fig. 5).

Effect of EGCG on cardiac fibrosis in diabetic rats. Quantitative analysis of the fibrotic region of the LV myocardium indicated an increased level of interstitial fibrosis in the hearts of the rats with STZ-induced diabetes after I/R injury compared to the 

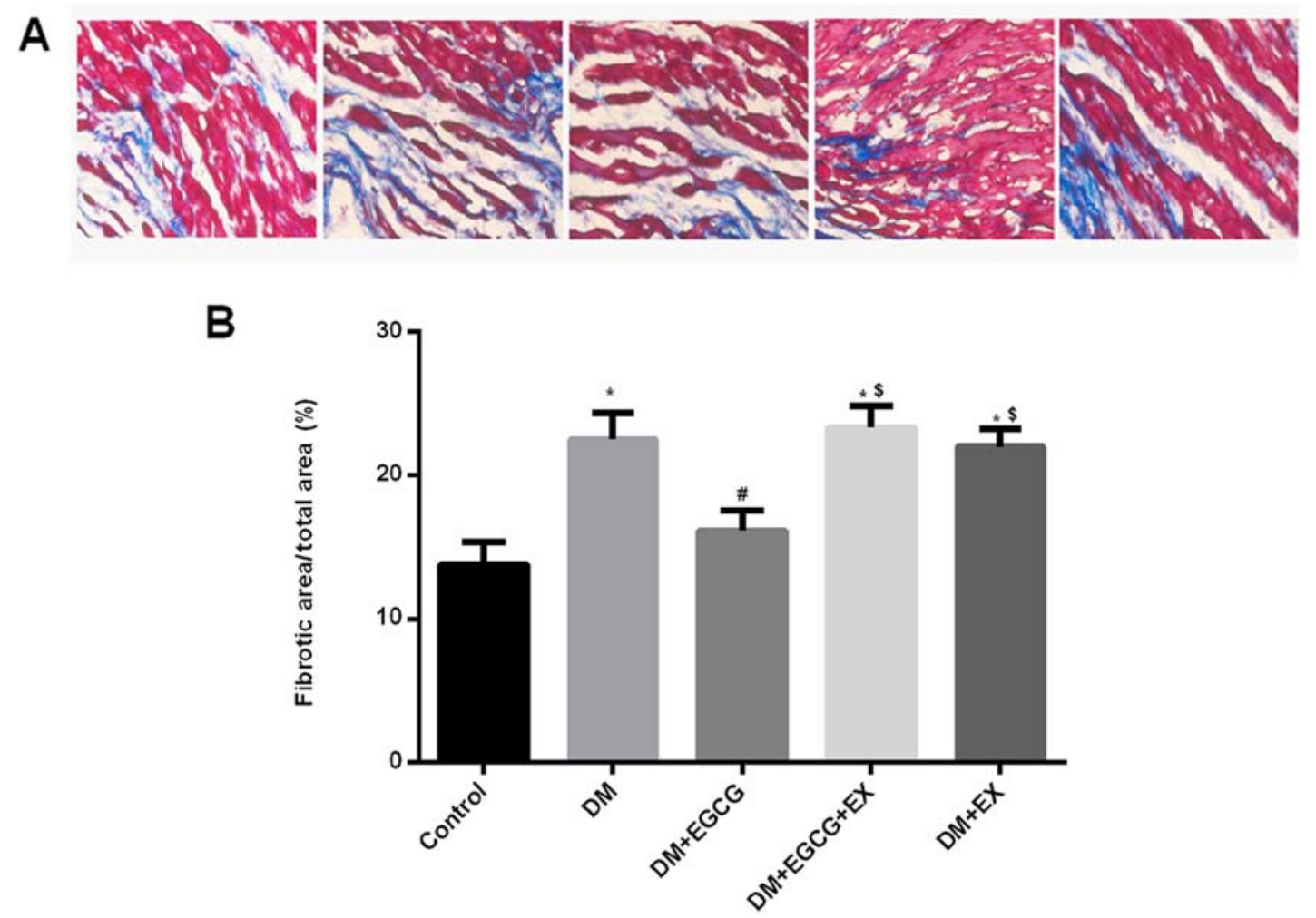

Figure 6. (-)-Epigallocatechin gallate (EGCG) treatment reduces the extent of cardiac fibrosis in the diabetic rats following ischemia/reperfusion (I/R) injury. (A) Masson trichrome-stained sections of left ventricles. (B) Quantification of cardiac fibrosis area from Masson trichrome-stained sections. Values are the means \pm SEM. $n=5$. ${ }^{*} \mathrm{P}<0.05$ vs. control group; ${ }^{\#} \mathrm{P}<0.05$ vs. DM group; ${ }^{\text {P }}<0.05$ vs. $\mathrm{DM}+\mathrm{EGCG}$ group.

control $(\mathrm{P}<0.05)$ (Fig. 6). However, EGCG treatment significantly reduced the extent of cardiac fibrosis compared with the diabetic rats $(\mathrm{P}<0.05)$. Additionally, the EGCG-induced reduction of cardiac fibrosis was abolished by co-treatment with EX527 in the diabetic rats $(\mathrm{P}<0.05)$.

Cellular apotosis, cell viability, LDH activity and MDA levels in $H / R$-injured $H 9 c 2$ cardiomyocytes. $\mathrm{H} / \mathrm{R}$ caused a marked increase in the apoptosis of the H9C2 cells compared to the normoxia groups $(\mathrm{P}<0.05)$ (Fig. 7). Exposure to $\mathrm{HG}+\mathrm{H} / \mathrm{R}$ induced an increase in the apoptotic index $(\mathrm{P}<0.05$, $\mathrm{HG}+\mathrm{H} / \mathrm{R}$ vs. $\mathrm{NG}+\mathrm{H} / \mathrm{R}$ group) and the administration of EGCG attenuated these changes (HG + EGCG vs. HG + H/R group). HG sensitized the cardiomyocytes to $\mathrm{H} / \mathrm{R}$ injury, which was manifested as reduced cardiomyocyte viability (Fig. 8A), and increased LDH (Fig. 8B) and MDA leels (Fig. 8C) (all $\mathrm{P}<0.05$ vs. $\mathrm{NG}+\mathrm{H} / \mathrm{R}$ group).

To confirm the role of SIRT1 in EGCG-mediated cellular protection and to explore the signaling pathway involved, we employed SIRT1 siRNA to specifically knockdown SIRT1 expression in H9c2 cells. Pre-treatment with EGCG markedly decreased the apoptosis to $25.5 \%$ and increased cell viability by $24.1 \%$ following $\mathrm{H} / \mathrm{R}(\mathrm{P}<0.05)$, compared with the $\mathrm{HG}+\mathrm{H} / \mathrm{R}$ group (Fig. 7). In addition, EGCG pre-treatment significantly reduced $\mathrm{LDH}$ activity and MDA formation by 16.7 and $23.6 \%$, respectively in the $\mathrm{H} / \mathrm{R}$-injured $\mathrm{H} 9 \mathrm{c} 2$ cardiomyocytes $(\mathrm{P}<0.05)$, compared to the $\mathrm{HG}+\mathrm{H} / \mathrm{R}$ group (Fig. $8 \mathrm{~B}$ and $\mathrm{C})$. However, the protective effects of EGCG pre-treatment were abrogated by transfection with SIRT1 siRNA $(\mathrm{P}<0.05)$, compared to EGCG treatment alone (Fig. 8).
Protein expression of SIRT1 and MnSOD in H9c2 cardiomyocytes subjected to $H / R$ injury. $\mathrm{HG}$ markedly decreased SIRT1 protein expression compared to that in the $\mathrm{NG}+\mathrm{H} / \mathrm{R}$ group $(\mathrm{P}<0.05)$ (Fig. 9). However, EGCG pre-treatment significantly increased the protein expression of SIRT1 and MnSOD (all $\mathrm{P}<0.05$ ), compared to the HG group (Fig. 10). Moreover, the effect of EGCG pre-treatment on the expression of these proteins was abolished by transfection with SIRT1 siRNA (all P<0.05), compared with EGCG pre-treatment alone (Fig. 10).

\section{Discussion}

This study provided evidence that rats with STZ-induced diabetes exhibited enhanced cardiac dysfunction, an increased myocardial infarct size, myocardial apoptosis and elevated oxidative stress, as well as cardiac fibrosis when they were subjected to myocardial I/R injury. However, these unfavorable outcomes were attenuated by EGCG administration. Additionally, HG aggravated cardiomyocyte apoptosis, reduced cellular viability and increased the oxidative stress induced by $\mathrm{H} / \mathrm{R}$ injury in $\mathrm{H} 9 \mathrm{c} 2$ cells, whereas EGCG protected the cardiomyocytes against $\mathrm{H} / \mathrm{R}$ impairment.

EGCG, as a polyphenol, possesses various pharmacological and biological properties and potentially exerts protective effects on the cardiovascular system (21). Previous studies have illustrated that EGCG alleviates myocardical I/R injury in nondiabetic rat hearts (22-25). However, these researchers have stated that further studies need to be performed using animal models of human disease such as diabetes, hypertension and 
A

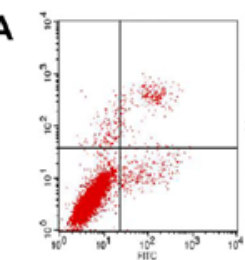

NG+Normaxia

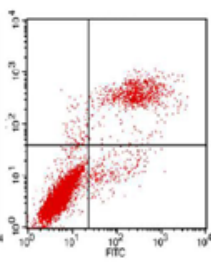

NG+HVR

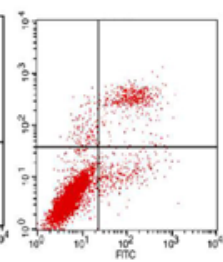

HG+Normaxia

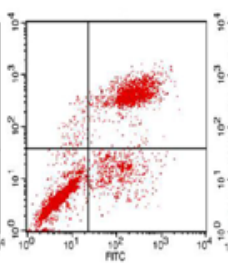

HG+H/R

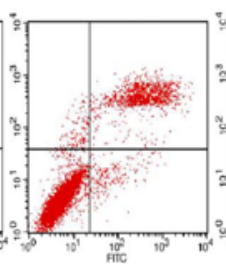

$H G+E G C G+H / R$

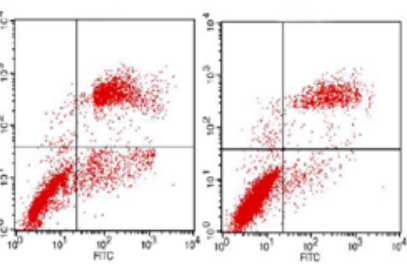

HG+EGCG+HR HG+EGCG+HVR +SIRT1 SiRNA +Scramble siRNA

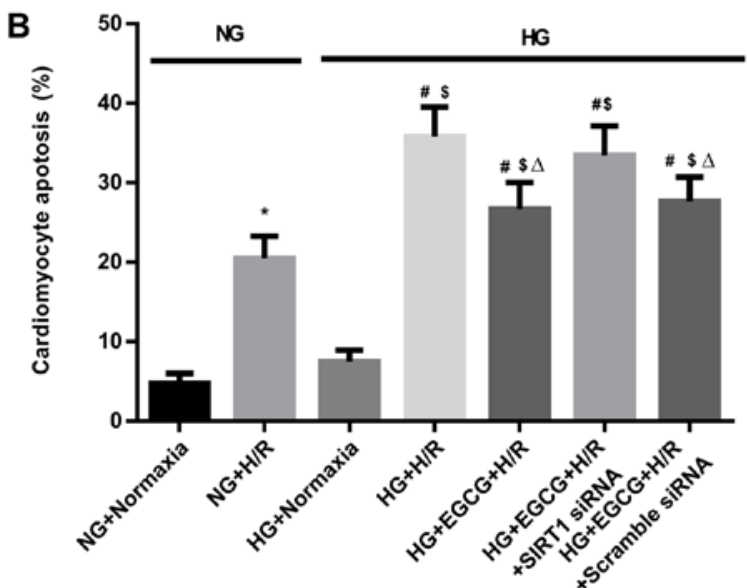

Figure 7. (-)-Epigallocatechin gallate (EGCG) treatment decreases cellular apoptosis. (A) Evaluation of cardiomyocyte apoptosis by flow cytometric analysis. (B) Quantification of cardiomyocyte apoptosis. Values are the means $\pm \mathrm{SEM}, \mathrm{n}=6 .{ }^{*} \mathrm{P}<0.05$ vs. normal glucose (NG) + normaxia group; ${ }^{\sharp} \mathrm{P}<0.05$ vs. high glucose $(\mathrm{HG})+$ normaxia group; ${ }^{\$} \mathrm{P}<0.05$ vs. $\mathrm{NG}+$ hypoxia/reoxygenation $(\mathrm{H} / \mathrm{R})$ group; ${ }^{\circ} \mathrm{P}<0.05$ vs. $\mathrm{HG}+\mathrm{H} / \mathrm{R}$ group.

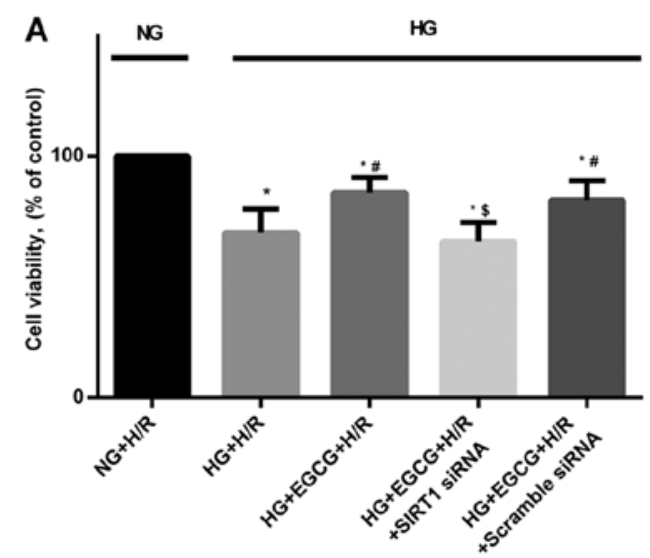

C
B

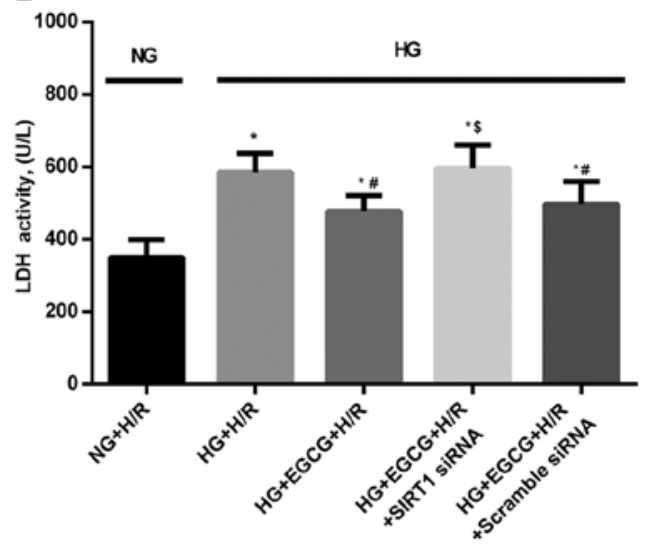

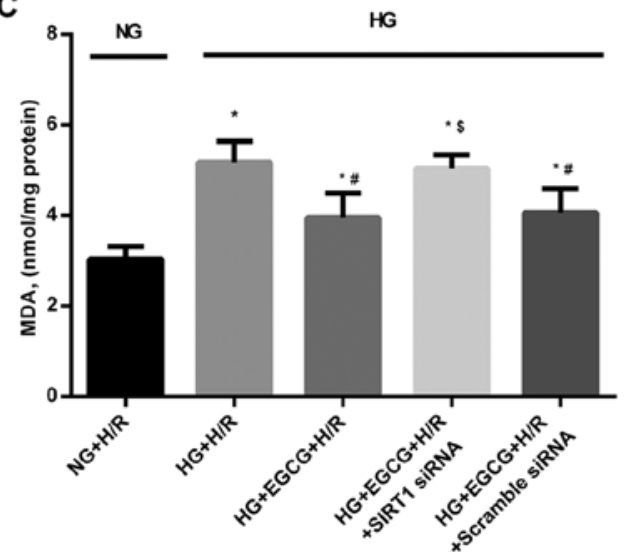

Figure 8. (-)-Epigallocatechin gallate (EGCG) treatment decreases lactate dehydrogenase (LDH) activity as well as malonaldehyde (MDA) levels and increases cell viability in hypoxia/reoxygenation (H/R)-injured H9c2 cardiomyocytes. (A) Relative cell viability in cardiomyocytes. (B) LDH activity in culture medium. (C) MDA production in the culture medium. Values are the means $\pm \mathrm{SEM}, \mathrm{n}=6$. ${ }^{\mathrm{P}} \mathrm{P}<0.05$ vs. normal glucose $(\mathrm{NG})+\mathrm{H} / \mathrm{R}$ group; $\mathrm{P}<0.05 \mathrm{vs}$. high glucose (HG) $+\mathrm{H} / \mathrm{R}$ group; ${ }^{\$} \mathrm{P}<0.05$ vs. $\mathrm{HG}+\mathrm{EGCG}+\mathrm{H} / \mathrm{R}$ group. 
A
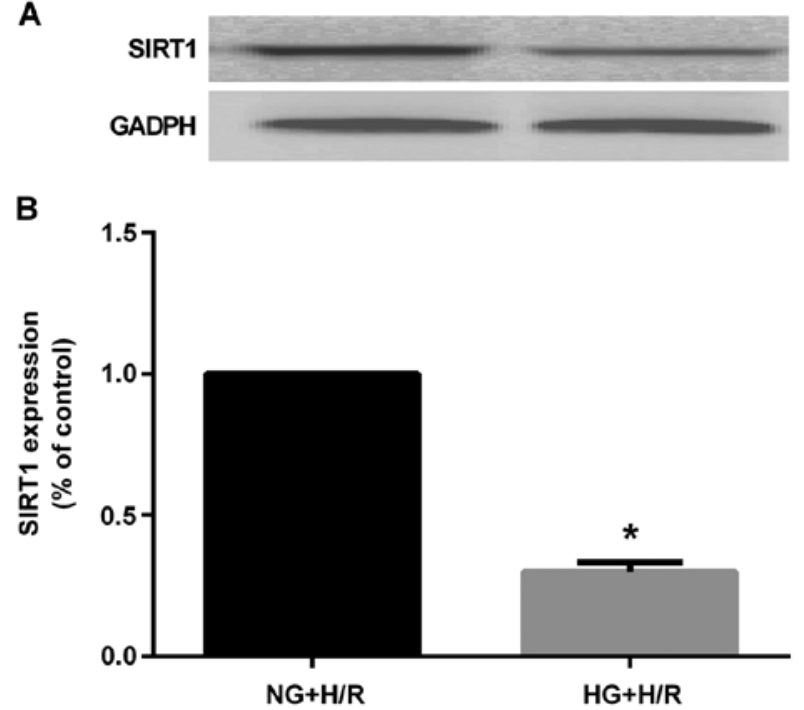

Figure 9. Silent information regulator 1 (SIRT1) protein expression in high glucose (HG) group and normal glucose (NG) group. (A) Representative western blots of SIRT1 protein in HG group and NG group; (B) Quantification of western blot data from (A). Values are the means \pm SEM, $n=6$. ${ }^{*} \mathrm{P}<0.05$ vs. $\mathrm{NG}+$ hypoxia/reoxygenation $(\mathrm{H} / \mathrm{R})$ group.

aging (22-26). Thus, the present study was conducted to further investigate the effect of EGCG on diabetic cardiomyopathy during myocardial I/R injury.

In the present study, all rats were fed with the AIN-93 diet without phytochemicals. The dosage of EGCG was determined based on a human dietary survey and animal studies $5 \mathrm{mg} / \mathrm{kg}$ body weight/day) of EGCG administration in rats has been extrapolated from a human study based on the average daily EGCG intake (27). However, most studies adopted EGCG to investigate its preventive and therapeutic effects on cardiovascular diseases in animal models ranging from 25 to $460 \mathrm{mg} / \mathrm{kg}$ body weight/day (17,19,28-30). The hepatotoxic effects of EGCG were observed when the daily oral dose was $1,500 \mathrm{mg} / \mathrm{kg}$ in mice (31), but there are no reports showing that daily EGCG intake at the dose less than $460 \mathrm{mg} / \mathrm{kg}$ body weight/day causes toxicity in rats. Based on the above evidence and our previous studies, we used $100 \mathrm{mg} / \mathrm{kg}$ body weight/day of EGCG in the present study.

There is substantial evidence to indicate that H9c2 cardiomyocytes cultured under HG can be used to explore the pathophysiology of diabetes $(32,33)$. The dose of EGCG that previous studies commonly adopted in $\mathrm{H} 9 \mathrm{c} 2$ cells ranged from 5 to $50 \mu \mathrm{M}(12,36,40)$. Although EGCG is poorly absorbed and its concentration in animal blood is lower than the dose $(20 \mu \mathrm{M})$ we adopted in the present study in vitro, Lambert et al observed that EGCG treatment at doses ranging from 20 to $600 \mu \mathrm{M}$ resulted in a linear increase in the cytosolic concentration of EGCG in human colon cancer cells (16). In fact, the beneficial effects of EGCG depend on the long-term cumulative action in the body (16). The protective effects of EGCG shown in the present study may not be observed in vitro experiments for such a short period of pre-treatment $(24 \mathrm{~h})$ if we use the subnanomolar concentration which is detectable in animal blood after absorption.
A
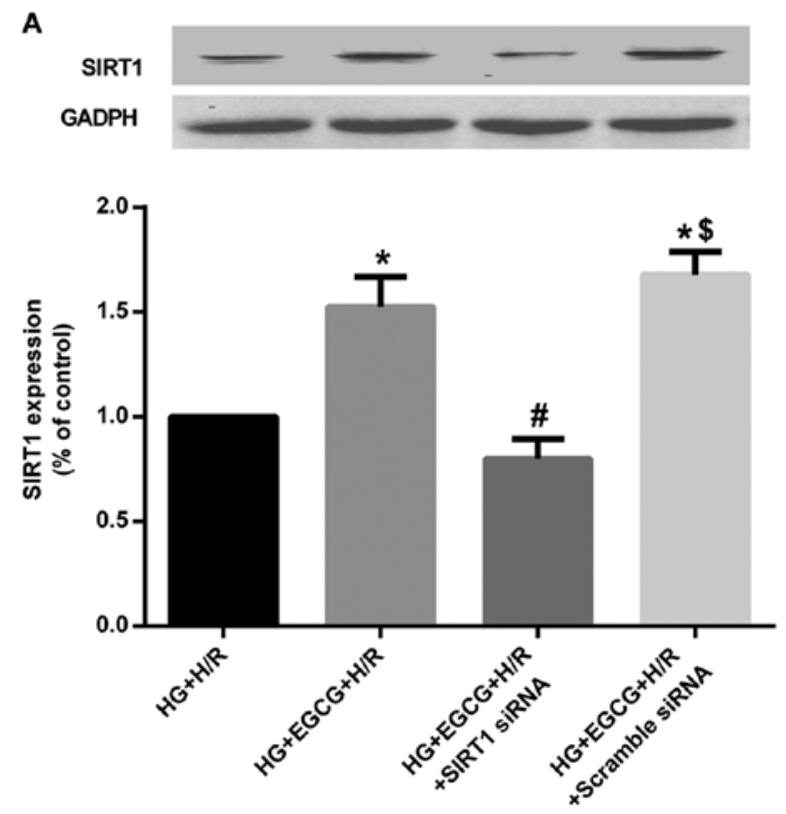

B
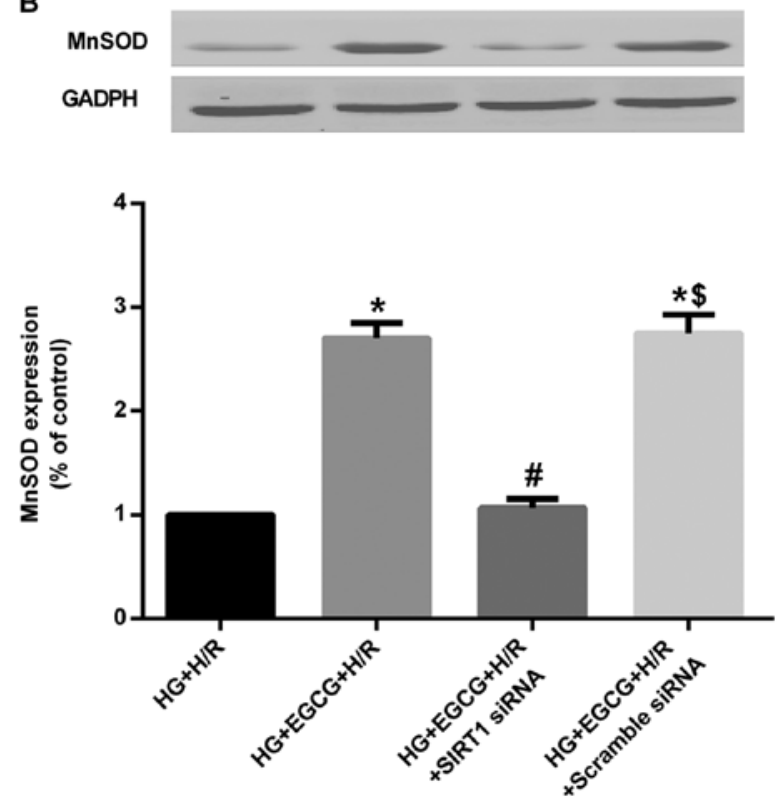

Figure 10. (-)-Epigallocatechin gallate (EGCG) pre-treatment enhances the protein expression of SIRT1 and manganese superoxide dismutase (MnSOD) in H9c2 cardiomyocytes subjected to hypoxia/reoxygenation (H/R)-injury. (A) Top panel: representative western blots of SIRT1 protein under the treatment of high glucose (HG), EGCG and/or SIRT1 siRNA. Bottom panel: quantification of western blot data from (A) top. (B) Top panel: representative western blots of MnSOD protein under the treatment of HG, EGCG and/or SIRT1 siRNA. Bottom panel: quantification of western blot data from (B) top. Values are the means $\pm \mathrm{SEM}, \mathrm{n}=6 .{ }^{*} \mathrm{P}<0.05 \mathrm{vs}$. HG $+\mathrm{H} / \mathrm{R}$ group; ${ }^{\#} \mathrm{P}<0.05$ vs. $\mathrm{HG}+\mathrm{EGCG}+\mathrm{H} / \mathrm{R}$ group; ${ }^{\$} \mathrm{P}<0.05$ vs. $\mathrm{HG}+\mathrm{EGCG}+\mathrm{H} / \mathrm{R}+\mathrm{SI}$ RT1 siRNA group.

It has previously been demonstrated that the myocardial protective effects of EGCG are primary related to its antioxidant properties (36). The intragastrical administration of EGCG was found to inhibit oxidative stress in rodent hearts (37). Hsieh et al also found that EGCG pre-treatment for $30 \mathrm{~min}$ protected $\mathrm{H} 9 \mathrm{c} 2$ cells from $\mathrm{H}_{2} \mathrm{O}_{2}$-induced oxidative stress (12). 
Similarly, we found that EGCG treatment reduced oxidative stress either in the hearts of the diabetic rats or in cardiomyocytes under hyperglycemic conditions after I/R injury.

It has been established that experimental diabetes aggravates $\mathrm{LV}$ dysfunction under conditions of I/R injury, as usually observed under clinical conditions. Hyperglycemia worsens cardiac performance and cell survival following myocardial $\mathrm{I} / \mathrm{R}$ injury via increased oxidative stress (36). Our data also indicated that the levels of oxidative damage indicators $\left(15-\mathrm{F}_{2 \mathrm{t}}\right.$ and MDA) were increased and cardiac dysfunction was manifested as a decrease in LVSP, $+\mathrm{dP} / \mathrm{dtmax}$ and $-\mathrm{dP} / \mathrm{dtmax}$ in I/R-injured diabetic rats, which is similar to the finding by $\mathrm{Xu}$ et al that rats with STZ-induced diabetes at at 8 weeks exhibited progressive abnormal cardiac systolic and diastolic function (2). Additionally, we also observed that EGCG improved cardiac performance, which was manifested as augmented LVSP, $+\mathrm{dP} /$ dtmax and -dP/dtmax.

Oxidative stress injury induced by the accumulation of ROS in diabetic hearts plays an important role in cardiac fibrosis (38). Our data demonstrated that oxidative damage indicators $\left(15-\mathrm{F}_{2 \mathrm{t}}\right.$ and MDA) and fibrosis were increased in diabetic rats after myocardial I/R injury, which was also in agreement with the findings of previous studies indicating that diabetes was associated with enhanced cardiac fibrosis after myocardial I/R injury $(34,35,39)$, which led to increased LV stiffness and decreased ventricular wall compliance, resulting in both systolic and diastolic dysfunction $(34,35)$. However, in the present study, EGCG pre-treatment reduced I/R injury-induced fibrosis in diabetic rats and attenuated adverse LV remodeling.

It has been reported that as a member of histone deacetylases III, SIRT1 plays a key role in cardioprotective effects during myocardial I/R injury $(9,33)$. These effects are mediated via the deacetylation of transcription factors and thye upregulation of antioxidant enzymes and other downstream gene targets $(9,29)$. Diabetic hearts are resistant to the myocardial infarct-limiting effects of ischemic preconditioning, accompanied with a marked inhibition of SIRT1 activity (7). These pathological alterations may diminish the ability of the heart to resist I/R injury. In the present study, we also observed that SIRT1 expression in the I/R-injured diabetic rats was $m$ markedly decreased compared to the normal rats, which paralleled with increased myocardial apoptosis, elevated oxidative stress and myocardial dysfunction in diabetic rats. Moreover, the decrement of SIRT1 triggered ROS generation and the impaired ROS clearance may thus aggravate diabetic myocardial I/R injury (41).

Although EGCG improves age-associated inflammation and oxidative stress in the liver by activating SIRT1 in healthy rats (42), few studies have examined the role of SIRT1 in regulating the effects of EGCG in ameliorating cardiac dysfunction, attenuating cardiomyocyte apoptosis and alleviating oxidative stress after myocardial I/R injury in diabetes. In the present study, we found that SIRT1 expression was significantly reduced in the hearts of diabetic rats and in $\mathrm{H} 9 \mathrm{c} 2$ cells under hyperglycemic conditions subjected to I/R injury. Moreover, EGCG upregulated the protein expression of SIRT1, and it improved cardiac dysfunction, ameliorated cardiomyocyte apoptosis and attenuated oxidative stress injury in cardiomyocytes of diabetic rats and in H9c2 cells after I/R injury under hyperglycemic conditions.
It has been demonstrated that SIRT1 can mediate protective effects against oxidative stress through the regulation of antioxidant genes, such as MnSOD (43). Likewise, we found that the EGCG-induced upregulation of SIRT1 protein expression also paralleled with an increase in MnSOD protein expression in diabetic cardiomyocytes in the setting of I/R injury. Moreover, the effects of EGCG on the protein expression of MnSOD were abolished by SIRT1 inhibitor, EX527, in vivo and SIRT1 siRNA in vitro. Therefore, EGCG may exert protective effects on diabetic hearts subjected to myocardial I/R injury by stimulating the SIRT1 signal. However, the precise role of SIRT1 in the EGCG-conferred cardioprotective effects under a diabetic state needs to be confirmed by further studies.

In conclusion, the present study demonstrated that EGCG attenuated cardiac dysfunction, reduced myocardial infarct size and cardiac fibrosis, and decreased myocardial apoptosis and oxidative stress by stimulating the SIRT1 signaling pathway in diabetic rats and in $\mathrm{H} 9 \mathrm{c} 2$ cells under hyperglycemic conditions when they were subjected to myocardial I/R injury. This suggests that EGCG may be a promising dietary supplementation for the attenuation or prevention of diabetic cardiomyopathy.

\section{Acknowledgements}

This study was supported by the National Natural Science Foundation of China (nos. 81201458, 81471844 and 81170768).

\section{References}

1. Donahoe SM, Stewart GC, McCabe CH, Mohanavelu S, Murphy SA, Cannon CP and Antman EM: Diabetes and mortality following acute coronary syndromes. JAMA 298: 765-775, 2007.

2. Xu J, Li H, Irwin MG, Xia ZY, Mao X, Lei S, Wong GT, Hung V, Cheung CW, Fang X, et al: Propofol ameliorates hyperglycemiainduced cardiac hypertrophy and dysfunction via heme oxygenase-1/signal transducer and activator of transcription 3 signaling pathway in rats. Crit Care Med 42: e583-e594, 2014.

3. Liu X, Wei J, Peng DH, Layne MD and Yet SF: Absence of heme oxygenase-1 exacerbates myocardial ischemia/reperfusion injury in diabetic mice. Diabetes 54: 778-784, 2005.

4. Koka S, Das A, Salloum FN and Kukreja RC: Phosphodiesterase-5 inhibitor tadalafil attenuates oxidative stress and protects against myocardial ischemia/reperfusion injury in type 2 diabetic mice. Free Radic Biol Med 60: 80-88, 2013.

5. Zheng Z, Chen H, Li J, Li T, Zheng B, Zheng Y, Jin H, He Y, $\mathrm{Gu} \mathrm{Q}$ and $\mathrm{Xu} X$ : Sirtuin 1-mediated cellular metabolic memory of high glucose via the LKB1/AMPK/ROS pathway and therapeutic effects of metformin. Diabetes 61: 217-228, 2012.

6. Nadtochiy SM, Yao H, McBurney MW, Gu W, Guarente L, Rahman I and Brookes PS: SIRT1-mediated acute cardioprotection. Am J Physiol Heart Circ Physiol 301: H1506-H1512, 2011.

7. Pillai VB, Sundaresan NR and Gupta MP: Regulation of Akt signaling by sirtuins: Its implication in cardiac hypertrophy and aging. Circ Res 114: 368-378, 2014.

8. Hori YS, Kuno A, Hosoda R and Horio Y: Regulation of FOXOs and p53 by SIRT1 modulators under oxidative stress. PLoS One 8: e73875, 2013.

9. Yang Y, Duan W, Li Y, Jin Z, Yan J, Yu S and Yi D: Novel role of silent information regulator 1 in myocardial ischemia. Circulation 128: 2232-2240, 2013.

10. Menegazzi M, Tedeschi E, Dussin D, De Prati AC, Cavalieri E, Mariotto S and Suzuki H: Anti-interferon gamma action of epigallocatechin-3-gallate mediated by specific inhibition of STAT1 activation. FASEB J 15: 1309-1311, 2001.

11. Townsend PA, Scarabelli TM, Pasini E, Gitti G, Menegazzi M, Suzuki H, Knight RA, Latchman DS and Stephanou A: Epigallocatechin-3-gallate inhibits STAT-1 activation and protects cardiac myocytes from ischemia/reperfusion-induced apoptosis. FASEB J 18: 1621-1623, 2004. 
12. Hsieh SR, Hsu CS, Lu CH, Chen WC, Chiu CH and Liou YM: Epigallocatechin-3-gallate-mediated cardioprotection by Akt/GSK-3 $3 /$ caveolin signalling in $\mathrm{H} 9 \mathrm{c} 2$ rat cardiomyoblasts. J Biomed Sci 20: 86, 2013.

13. Sulaiman M, Matta MJ, Sunderesan NR, Gupta MP, Periasamy M and Gupta M: Resveratrol, an activator of SIRT1, upregulates sarcoplasmic calcium ATPase and improves cardiac function in diabetic cardiomyopathy. Am J Physiol Heart Circ Physiol 298 : H833-H843, 2010.

14. Queen BL and Tollefsbol TO: Polyphenols and aging. Curr Aging Sci 3: 34-42, 2010.

15. Li H, Yao W, Irwin MG, Wang T, Wang S, Zhang L and Xia Z: Adiponectin ameliorates hyperglycemia-induced cardiac hypertrophy and dysfunction by concomitantly activating Nrf2 and Brg1. Free Radic Biol Med 84: 311-321, 2015.

16. Lambert JD, Lee MJ, Diamond L, Ju J, Hong J, Bose M, Newmark HL and Yang CS: Dose-dependent levels of epigallocatechin-3-gallate in human colon cancer cells and mouse plasma and tissues. Drug Metab Dispos 34: 8-11, 2006.

17. Hsieh SR, Tsai DC, Chen JY, Tsai SW and Liou YM: Green tea extract protects rats against myocardial infarction associated with left anterior descending coronary artery ligation. Pflugers Arch 458: 631-642, 2009.

18. Yu L, Sun Y, Cheng L, Jin Z, Yang Y, Zhai M, Pei H, Wang X, Zhang H, Meng Q, et al: Melatonin receptor-mediated protection against myocardial ischemia/reperfusion injury: Role of SIRT1. J Pineal Res 57: 228-238, 2014.

19. Yan J, Feng Z, Liu J, Shen W, Wang Y, Wertz K, Weber P, Long $\mathbf{J}$ and Liu J: Enhanced autophagy plays a cardinal role in mitochondrial dysfunction in type 2 diabetic Goto-Kakizaki (GK) rats: Ameliorating effects of (-)-epigallocatechin-3-gallate. J Nutr Biochem 23: 716-724, 2012.

20. Wu Y, Xia ZY, Dou J, Zhang L, Xu JJ, Zhao B, Lei S and Liu HM: Protective effect of ginsenoside Rb1 against myocardial ischemia/reperfusion injury in streptozotocin-induced diabetic rats. Mol Biol Rep 38: 4327-4335, 2011.

21. Yamazaki KG, Romero-Perez D, Barraza-Hidalgo M, Cruz M Rivas M, Cortez-Gomez B, Ceballos G and Villarreal F: Short- and long-term effects of (-)-epicatechin on myocardial ischemia-reperfusion injury. Am J Physiol Heart Circ Physiol 295: H761-H767, 2008.

22. Yanagi S, Matsumura K, Marui A, Morishima M, Hyon SH, Ikeda T and Sakata R: Oral pretreatment with a green tea polyphenol for cardioprotection against ischemia-reperfusion injury in an isolated rat heart model. J Thorac Cardiovasc Surg 141: 511-517, 2011.

23. Kim CJ, Kim JM, Lee SR, Jang YH, Kim JH and Chun KJ: Polyphenol (-)-epigallocatechin gallate targeting myocardial reperfusion limits infarct size and improves cardiac function. Korean J Anesthesiol 58: 169-175, 2010

24. Piao CS, Kim DS, Ha KC, Kim HR, Chae HJ and Chae SW: The protective effect of epigallocatechin-3 gallate on ischemia/reperfusion injury in isolated rat hearts: An ex vivo approach. Korean J Physiol Pharmacol 15: 259-266, 2011.

25. Kim SJ, Li M, Jeong CW, Bae HB, Kwak SH, Lee SH, Lee HJ, Heo BH, Yook KB and Yoo KY: Epigallocatechin-3-gallate, a green tea catechin, protects the heart against regional ischemiareperfusion injuries through activation of RISK survival pathways in rats. Arch Pharm Res 37: 1079-1085, 2014.

26. Lejay A, Fang F, John R, Van JA, Barr M, Thaveau F, Chakfe N, Geny B and Scholey JW: Ischemia reperfusion injury, ischemic conditioning and diabetes mellitus. J Mol Cell Cardiol 91: 11-22, 2016.

27. Arts IC, Hollman PC, Feskens EJ, Bueno de Mesquita HB and Kromhout D: Catechin intake and associated dietary and lifestyle factors in a representative sample of Dutch men and women. Eur J Clin Nutr 55: 76-81, 2001.
28. Liu TT, Liang NS, Li Y, Yang F, Lu Y, Meng ZQ and Zhang LS: Effects of long-term tea polyphenols consumption on hepatic microsomal drug-metabolizing enzymes and liver function in Wistar rats. World J Gastroenterol 9: 2742-2744, 2003.

29. Shi Z, Fu F, Yu L, Xing W, Su F, Liang X, Tie R, Ji L, Zhu M, Yu J, et al: Vasonatrin peptide attenuates myocardial ischemia-reperfusion injury in diabetic rats and underlying mechanisms. Am J Physiol Heart Circ Physiol 308: H281-H290, 2015.

30. Niu Y, Na L, Feng R, Gong L, Zhao Y, Li Q, Li Y and Sun C: The phytochemical, EGCG, extends lifespan by reducing liver and kidney function damage and improving age-associated inflammation and oxidative stress in healthy rats. Aging Cell 12: 1041-1049, 2013

31. Lambert JD, Kennett MJ, Sang S, Reuhl KR, Ju J and Yang CS: Hepatotoxicity of high oral dose (-)-epigallocatechin-3-gallate in mice. Food Chem Toxicol 48: 409-416, 2010.

32. Xue R, Lei S, Xia ZY, Wu Y, Meng Q, Zhan L, Su W, Liu H, Xu J, Liu Z, et al: Selective inhibition of PTEN preserves ischaemic post-conditioning cardioprotection in STZ-induced type 1 diabetic rats: Role of the PI3K/Akt and JAK2/STAT3 pathways. Clin Sci (Lond) 130: 377-392, 2016.

33. Yu L, Liang H, Dong X, Zhao G, Jin Z, Zhai M, Yang Y, Chen W, Liu J, Yi W, et al: Reduced silent information regulator 1 signaling exacerbates myocardial ischemia-reperfusion injury in type 2 diabetic rats and the protective effect of melatonin. J Pineal Res 59: 376-390, 2015.

34. Wu R, Smeele KM, Wyatt E, Ichikawa Y, Eerbeek O, Sun L, Chawla K, Hollmann MW, Nagpal V, Heikkinen S, et al: Reduction in hexokinase II levels results in decreased cardiac function and altered remodeling after ischemia/reperfusion injury. Circ Res 108: 60-69, 2011.

35. Toldo S, Breckenridge DG, Mezzaroma E, Van Tassell BW, Shryock J, Kannan H, Phan D, Budas G, Farkas D, Lesnefsky E, et al: Inhibition of apoptosis signal-regulating kinase 1 reduces myocardial ischemia-reperfusion injury in the mouse. J Am Heart Assoc 1: e002360, 2012

36. Chen WC, Hsieh SR, Chiu CH, Hsu BD and Liou YM: Molecular identification for epigallocatechin-3-gallate-mediated antioxidant intervention on the $\mathrm{H}_{2} \mathrm{O}_{2}$-induced oxidative stress in H9c2 rat cardiomyoblasts. J Biomed Sci 21: 56, 2014.

37. Li H, Bian Y, Zhang N, Guo J, Wang C, Lau WB and Xiao C: Intermedin protects against myocardial ischemia-reperfusion injury in diabetic rats. Cardiovasc Diabetol 12: 91, 2013.

38. Wang B, Yang Q, Bai WW, Xing YF, Lu XT, Sun YY and Zhao YX: Tongxinluo protects against pressure overload-induced heart failure in mice involving VEGF/Akt/eNOS pathway activation. PLoS One 9: e98047, 2014.

39. Eguchi M, Kim YH, Kang KW, Shim CY, Jang Y, Dorval T, Kim KJ and Sweeney G: Ischemia-reperfusion injury leads to distinct temporal cardiac remodeling in normal versus diabetic mice. PLoS One 7: e30450, 2012.

40. Liu J, Tang Y, Feng Z, Hou C, Wang H, Yan J, Liu J, Shen W, Zang W, Liu J, et al: Acetylated FoxO1 mediates high-glucose induced autophagy in $\mathrm{H} 9 \mathrm{c} 2$ cardiomyoblasts: Regulation by a polyphenol -(-)-epigallocatechin-3-gallate. Metabolism 63: 1314-1323, 2014

41. Alcendor RR, Gao S, Zhai P, Zablocki D, Holle E, Yu X, Tian B, Wagner T, Vatner SF and Sadoshima J: Sirt1 regulates aging and resistance to oxidative stress in the heart. Circ Res 100: 1512-1521, 2007.

42. Puthanveetil P, Wan A and Rodrigues B: FoxO1 is crucial for sustaining cardiomyocyte metabolism and cell survival. Cardiovasc Res 97: 393-403, 2013.

43. Salminen A, Kaarniranta K and Kauppinen A: Crosstalk between oxidative stress and SIRT1: Impact on the aging process. Int J Mol Sci 14: 3834-3859, 2013 